\title{
Hyperchaos and Bifurcations in a Driven Van der Pol-Duffing Oscillator Circuit
}

\author{
U. E. Vincent - B. R. Nana Nbendjo • A. A. Ajayi • A. N. Njah • P. V. \\ E. McClintock
}

Received: 28 May, 2014 / Revised: 12 July, 2014 / Accepted: 16th July 2014

\begin{abstract}
We investigate the dynamics of a driven Van der Pol-Duffing oscillator circuit and show the existence of higher-dimensional chaotic orbits (or hyperchaos), transient chaos, strange-nonchaotic attractors, as well as quasiperiodic orbits born from Hopf bifurcating orbits. By computing all the Lyapunov exponent spectra, scanning a wide range of the driving frequency and driving amplitude parameter space, we explore in twoparameter space the regimes of different dynamical behaviours.
\end{abstract}

Keywords Hyperchaos - Transient Chaos - Chaos · Strange attractors · driven van der-Duffing oscillators

\section{U. E. Vincent}

Permanent address:Department of Physical Sciences, Redeemer's University, Redemption City, Nigeria.

Department of Physics, Lancaster University, Lancaster LA1 4YB, United Kingdom. E-mail: u.vincent@lancaster.ac.uk; ue_vincent@yahoo.com

B. R Nana-Nbendjo

Laboratory of Modeling and Simulation in Engineering, Biomimetism and Prototypes, Faculty of Sciences,

University of Yaounde I, Yaounde, Cameroon.

A. A. Ajayi

Department of Physics, University of Agriculture Abeokuta, P.M.B. 2240, Abeokuta, Nigeria.

Department of Physics, Federal College of Education, Akoka,

Yaba, Lagos, Nigeria.

E-mail: ayotundeaj@yahoo.com

A. N. Njah

Department of Physics, University of Lagos, Akoka, Lagos, Nigeria

P. V. E. McClintock

Department of Physics, Lancaster University, Lancaster LA1 4YB, United Kingdom.

\section{Introduction}

Electronic circuits exhibiting chaotic behaviour have attracted wide attention because they provide excellent platform for practical implementation of chaotic oscillators (See for example Ref. [5, 19, 13, 24, 28, 8, 2, 25,39, 35, $7,11,23,10,9,30,38]$ and references therein). Among the several chaotic circuits, the Van der-Pol Duffing oscillator is a very prominent and important classical model circuit that has been extensively studied in the context of several specific problems ranging from global bifurcation structures, control and synchronization (see for example Ref. [19,13,11,10,9,41]).

The Van der Pol-Duffing oscillator can be used as a model in physics, engineering, electronics, biology, neurology and many other disciplines [20,18]. King et al. $[19,13]$ proposed a schematic circuit of Van derPol Duffing oscillator which is equivalent to Chua's autonomous circuit but with a cubic nonlinear element [5] and can be described by the following set of autonomous dimensionless differential equations:

$\dot{x}=-m\left(x^{3}-\alpha x-y+\mu_{o f}\right), \dot{y}=x-y-z, \dot{z}=\beta y(1)$

where the overdots stands for the differentiation with respect to normalized time $\tau ; x, y$ and $z$ corresponds to the re-scaled form of the voltages across $C_{1}, C_{2}$ and the current through $L$ respectively (1). $\alpha, \beta$ and $\mu$ are the parameters of the system. System (1) exhibits rich dynamical behaviours including a double-scroll chaos for the system parameters: $\alpha=0.35, \beta=300, \mu_{o f}=0$, and $m=100$ [19,13].

In 2005 and 2007, Fotsin et al. [11,10,9] proposed a modified Van der Pol-Duffing oscillator (MVDPD) circuit by adding a series resistance of the inductor $R_{L}$, thus yielding the dimensionless equation:

$\dot{x}=-m\left(x^{3}-\alpha x-y+\mu_{o f}\right), \dot{y}=x-y-z, \dot{z}=\beta y-\gamma z(2)$ 
where the additional parameter, $\gamma$ is associated with the effect of the resistance of the inductor on the system dynamics. With $\gamma=0.2$, system (2) exhibits the same double-scroll attractor similar to system (1) and when $\mu_{o f} \neq 0$, a one scroll chaotic attractor was found [9]. Recently, Matouk and Agiza [27] introduced another modification by adding to the Van der-Pol Duffing oscillator circuit described by the autonomous system (1) a resistor in parallel with the inductor, giving rise to a new system which takes the form:

$\dot{x}=-m\left(x^{3}-\alpha x-y\right), \dot{y}=x-\mu y-z, \dot{z}=\beta y$.

The modified circuit by Matouk and Agiza [27] has some advantages in that all the dynamics of the oscillator are displayed in a small range by varying the new systems parameter, $\mu$ arising from the parallel resistance. Furthermore, a complete description of the regions in the parameter space for which multiple small periodic solutions arise through the Hopf bifurcations at the equilibria in system (3) was recently analyzed in detail by Braga et al. [3]. Very recently, a similar work was done for the Chua's circuit by Prebianca et al. [30].

All of the above Van der-Pol Duffing oscillator circuit models deal with self-oscillatory (i. e. autonomous) systems. In this paper, we investigate the dynamics of a nonautonomous unified Van der-Pol Duffing oscillator (UVDP) circuit by introducing a changeable electrical power source (acting as subject to a periodic driving force) [23]. We examine the combined effects of the series and parallel resistances as shown in Figure 1 , as well as the periodic driving. Systems with periodic forcing are often used for practical applications, in areas such as communications. Much effort has also been keenly devoted to understanding the dynamics of nonautonomous oscillators driven from equilibrium by a variety of external forcing because deterministic influences often arise in practice as in cellular dynamics, blood circulation, and brain dynamics $[37,36]$. The model we investigate exhibit more complex and richer dynamics as the amplitude and frequency of the forcing is varied. Besides various chaotic and periodic orbits, our system also show chaos-hyperchaos transitions, coexisting attractors, as well as Hopf bifurcations in which quasiperiodic orbits are born. We first present in section 2, the unified and periodically forced Van der PolDuffing oscillator (UVDP) and discuss its basic dynamical properties. Sections 3 and 4 are devoted to the local and global bifurcation structures and chaotic behaviour respectively; while section 5 concludes the paper.

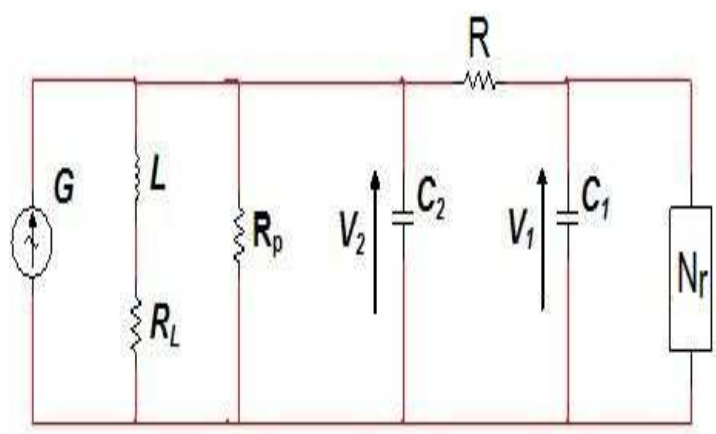

Fig. 1 Driven Van der Pol-Duffing oscillator circuit.

\section{Description of the Model}

\subsection{Driven Van der Pol-Duffing oscillator Circuit}

The periodically driven Van der Pol-Duffing oscillator considered here can be modeled by the circuit shown in Figure 1, in which a series resistance $R$ is added to the $C_{2}$ branch of the circuit, while another resistance $R_{p}$ is placed parallel to it. In addition, a periodic signal generator $G$, acting as periodic driving force is connected to the left end of the circuit as shown. By applying Kirchhoff's laws to the various branches of the circuit of Figure 1, and noting that the $i(v)$ characteristics of the nonlinear resistor $(\mathrm{N})$ is approximated by the cubic polynomial $i\left(V_{1}\right)=a V_{1}+b V_{1}^{3},(a<0, b>0)$ [27], we obtain the following set of equations:

$$
\begin{aligned}
\frac{d V_{1}}{d t} & =-\frac{1}{C_{1}}\left(\frac{1}{R}+a\right) V_{1}+\frac{V_{2}}{R C_{1}}-\frac{b}{C_{1}} V_{1}^{3}, \\
\frac{d V_{2}}{d t} & =\frac{V_{1}}{R C_{2}}-\frac{\mu V_{2}}{R C_{2}}-\frac{i_{L}}{C_{2}}+\frac{i_{G}}{C_{2}} \sin \omega t, \\
\frac{d i_{L}}{d t} & =\frac{V_{2}}{L}-\frac{R_{L}}{L} i_{L} .
\end{aligned}
$$

Making appropriate rescaling of eq. (4) by setting $x=V_{1} \sqrt{b R}, y=V_{2} \sqrt{b R}, z=i_{L} \sqrt{b R^{3}}, \tau=\frac{t}{R C_{2}}$, $m=\frac{C_{2}}{C_{1}}, \alpha=-(1+a R), \beta=\frac{R^{2} C_{2}}{L}, \gamma=\frac{R_{L} R C_{2}}{L}$, $a_{0}=R(\sqrt{b R}) I_{G}, \Omega=\frac{\omega}{R C_{2}}$ and $\mu=\frac{R+R_{p}}{R_{p}}$, we obtain the following dimensionless equation:

$\dot{x}=-m\left(x^{3}-\alpha x-y\right)$,

$\dot{y}=x-\mu y-z+a_{0} \sin \omega \tau$,

$\dot{z}=\beta y-\gamma z$.

System (5) is a three-dimensional nonautonomous system; where the new parameters $\omega$ and $a_{0}$ are the frequency and amplitude of the periodic driving force, respectively. Thus, our model system (5) has four Lyapunov exponents, allowing for hyperchaotic behaviour, 
i.e. with the possibility of having two positive Lyapunov exponents along with one zero and one negative. Beginning with the pioneering work of Rössler [31], the study of hyperchaos has witnessed tremendous research interest in the last three decades, in the fields of nonlinear circuits $[23,31,43,4]$, secure communications [1,34,21], nonlinear optics [16], control and synchronization [37, $6,44]$, to mention a few. Due to its great potential in technological applications, the generation of hyperchaos from nonlinear systems has recently become one focal topic for research $[23,37,22,33]$. Our goal here is to investigate the effect of $\omega$ and $a_{0}$ on the dynamics of (5) and show that under periodic driving, the modified Van der Pol-Duffing oscillator would exhibit richer dynamical complexities, including the existence of hyperchaos.

\subsection{Equilibria and their stability}

To examine the stability of system (5), we first obtain the fixed points by solving the general equation $F(\dot{u})=$ 0 , where $F$ is the nullcline and $u$ is the vector space containing $x, y$ and $z$. It appears that for $\alpha(\mu \gamma+\beta)+$ $\gamma<0$, we have only one fixed point $S_{0}(0,0,0)$ and, for $\alpha(\mu \gamma+\beta)+\gamma>0$, we have three fixed points, namely $S_{1}(-p,-\gamma q,-\beta q), S_{0}(0,0,0)$ and $S_{2}(p, \gamma q, \beta q)$ where

$p=\sqrt{\frac{\alpha(\mu \gamma+\beta)+\gamma}{\mu \gamma+\beta}} ; q=\sqrt{\frac{\alpha(\mu \gamma+\beta)+\gamma}{(\mu \gamma+\beta)^{3}}}$.

In both cases, by considering the Jacobian matrix of one of these equilibria and calculating their eigenvalues, we can investigate the stability of the equilibrium point based on the roots of the characteristic equation

$S^{3}+b_{0} S^{2}+b_{1} S+b_{2}=0$

where $b_{0}=\mu+\gamma-m\left(3 x_{0}^{2}-\alpha\right), b_{1}=\mu \gamma+\beta+m\left(3 x_{0}^{2}-\right.$ $\alpha)(\mu+\gamma)-m$, and $b_{2}=-m\left(3 x_{0}^{2}-\alpha\right)(\mu \gamma+\beta)+m \gamma$.

We know that the fixed points are stable if the real parts of the roots of the characteristics equation are all negative. Otherwise, the fixed points are unstable. Using Routh-Hurwitz criterion [17], for the sign of the real part of the roots, we obtain that the real parts of the roots are negative if and only if all the other coefficients $b_{0}, b_{1}$ and $b_{2}$ are positive and all the determinants $\Delta_{1}=b_{0} b_{2}-b_{1}$ and $\Delta_{2}=b_{2}\left(b_{0} b_{2}-b_{1}\right)$ also positive. Before analyzing the stability status of each point, it is important to consider the practical process of modeling system 4 (see section 2.1). It is clear that only $\alpha$ can take on negative or positive values. The other parameters are always positive. Focussing on the fixed point $S_{0}$, the analysis leads us to the conclusion that for $0<\alpha<\frac{\mu+\gamma}{m}$ this fixed point is always stable. The other fixed points $S_{1}$ and $S_{2}$ can be stable or unstable depending on the choice of space parameter of the system. Therefore the stability condition should be checked according to the criteria defined above before any use is made of the system.

\section{Local Bifurcations and Attractors}

The numerical results that follows were performed using the standard fourth order Runge-Kutta routine with step-size $h=2 \pi /(N \omega)$, where $N=100$ is an integer. The Lyapunov exponents were computed using the Wolf et al.'s algorithm [42] which requires the linearized version of equation (5) for small variations from the trajectory in tangent space and the Graham Smith's orthonormalization procedure allows us to compute the complete spectrum from maximal to minimal. For clarity and brevity, we will present only the first two exponents, namely $\lambda_{1}$ and $\lambda_{2}$ which determine exclusively the system's behaviours; while the full spectra would be considered in analyzing the global bifurcation in parameter phase space. Unless otherwise stated, the following parameters were fixed: $\alpha=0.35, \beta=300$, and $m=100$; while the other parameters, namely $\mu, \gamma, a_{0}$ and $\omega$ were varied for the different cases considered. In the absence of the forcing we obtain the Fotsin and Woafo model when $\mu=1.0$, and $\gamma=0.2[11,10]$; whereas, when $\mu>1.0$ and $\gamma=0$ we have the Matouk and Agiza model [27].

We begin by considering the case for $\mu=1.0$ and $\gamma=0.2$. We set $\omega=10$ and display in Fig. 2 the bifurcation diagram as function of the driving amplitude $a_{0}$ (upper panel (a)) and the corresponding maximal $\left(\lambda_{1}\right)$ and second $\left(\lambda_{2}\right)$ Lyapunov exponents in the same range of $a_{0}$ (lower panel (b)). Clearly, we observe four distinct regimes corresponding to different transitions, and denoted by I, II, III and IV. Notice that when $a_{0}=0$, a double-scroll chaotic attractor exist $[11,10]$ for the chosen parameters. However, when $a_{0}$ increases gradually, the chaotic attractor loses it stability and undergoes a variety of bifurcations from regions (I) to (IV) which is terminated in a controlled systems of period-1 orbit. In particular, regimes I and III show several interesting dynamical transitions including the existence of hyperchaotic solution which we will describe below.

In Fig. 3, we display a zoom of the Lyapunov exponents corresponding to region I of Fig. 2. The first (maximal) Lyapunov exponent, $\lambda_{1}$ is positive for $a_{0} \leq$ 1.8 and drops to zero at $a_{0} \approx 1.8$. The second Lyapunov exponent $\lambda_{2}$ oscillates marginally around zero for $a_{0}<0.5$, positive for $0.5 \leq a_{0} \leq 1.57$ and for $a_{0}>$ 1.57 , drops to negative. Obviously, the system has two positive Lyapunov exponents in the amplitude interval $0.4 \leq a_{0} \leq 1.6$, indicating instability in two directions and signalling hyperchaotic state [31]; one positive and 


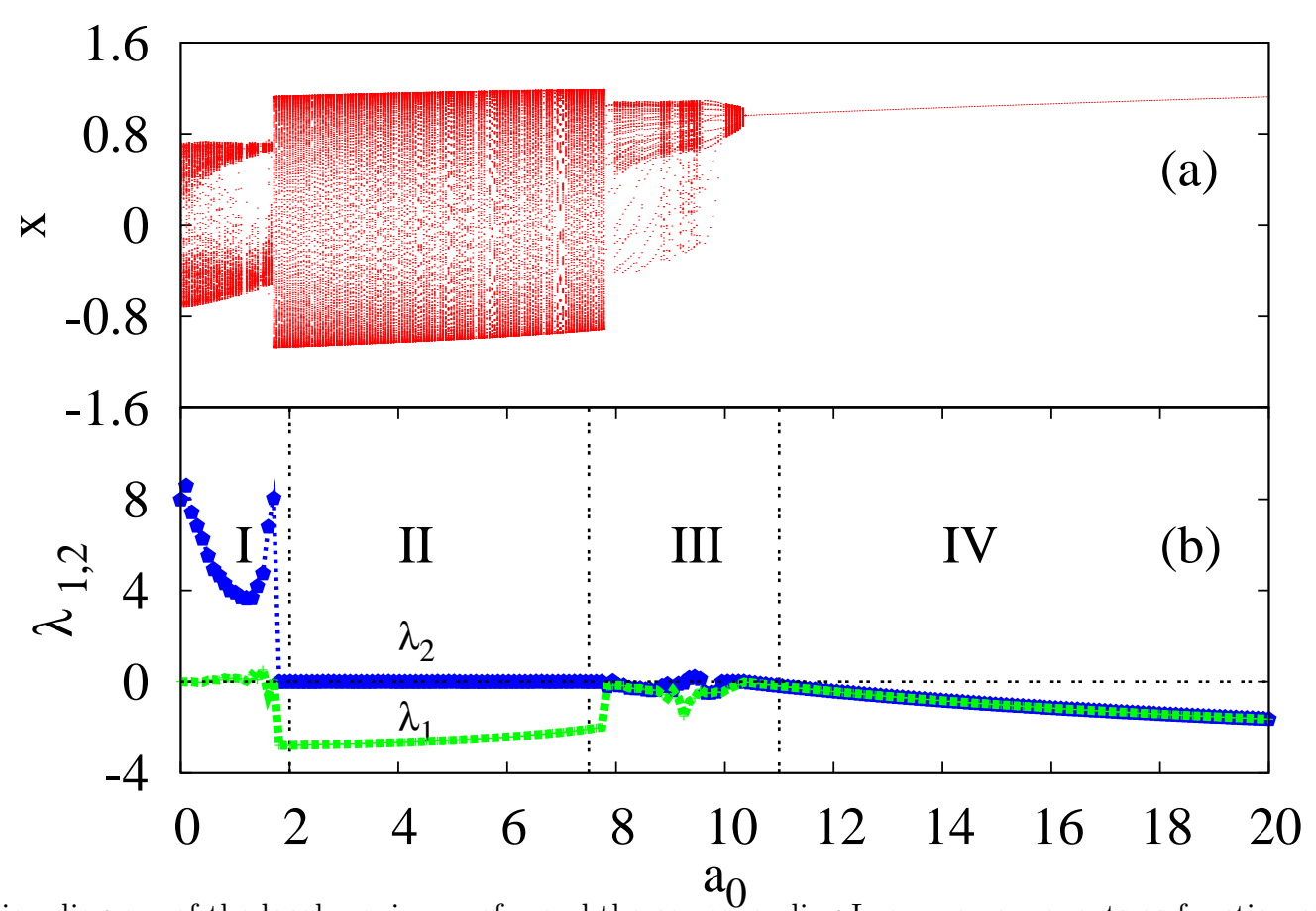

Fig. 2 Bifurcation diagram of the local maximum of $x$ and the corresponding Lyapunov exponents as function of the driving amplitude, $a_{0} . \alpha=0.35, \beta=300, m=100, \gamma=0.2, \mu=1.0$, and $\omega=10$. In (b), solid line denotes the first Lyapunov exponents, while dotted line denotes the second Lyapunov exponents.

one negative Lyapunov exponents for $1.6 \leq a_{0} \leq 1.8$ (chaos); and one zero and one negative Lyapunov exponents for $1.8 \leq a_{0} \leq 7.8$-a signature of quasiperiodicity. The existence of hyperchaotic behaviour implies that the system exhibits anomalous instability. Thus, a change in $a_{0}$ to a value within this regime switches the system from chaos $\rightarrow$ hyperchaos $\rightarrow$ chaos $\rightarrow$ quasiperiodicity (a limit cycle) for the same orbit as the system transits from region I to region II.

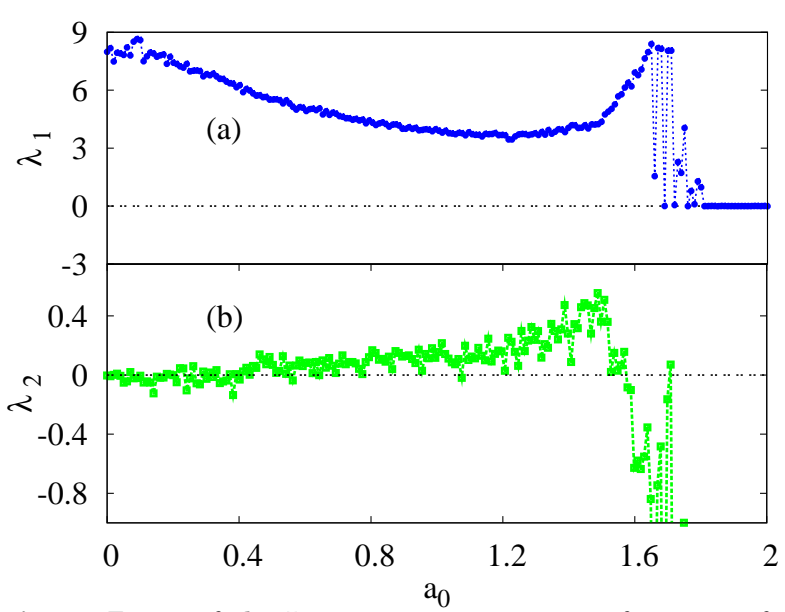

Fig. 3 Zoom of the Lyapunov exponents as function of the driving amplitude, for region I in Figure 2 for the same set of parameters.
Apparently, the chaos-hyperchaos transition at $a_{0} \approx$ 0.4 is accompanied by the so-called attractor splitting (or reverse attractor merging) crises earlier reported by Grebogi et al. [14,15] and well classified by Ott [29]. Here, the double-scroll chaotic attractor, corresponding to the unforced system, and shown in Fig. 4(a) breaks up into two branches, which eventually merge as $a_{0}$ further increases during the hyperchaos-chaos transition, forming another structurally different chaotic attractor. The $a_{0}$ range for which two branches of chaotic band exist in the bifurcation structure corresponds to the hyperchaotic regime. Our detailed numerical simulations show that the hyperchaotic behaviour in the forced system arises due to attractor splitting crises, though the branching point depends on the value of $\omega$. Furthermore, we observed that the chaos-quasiperiodic transition is accompanied by boundary crisis at $a_{0}>$ $a_{0}^{c} \approx 1.762$ in which a chaotic attractor collides with an unstable periodic orbit on its basin boundary [14, $15,29,40]$; and consequently replaced by a chaotic transient, wherein the orbit spends a very long time in the neighbourhood of the non-attracting chaotic set before leaving; and thereafter moves on to the stable quasiperiodic state that governs its long-time motion. Fig. 5 shows a typical chaotic transient motion for $a_{0}=1.77$. During the initial phase $(t \leq 580)$, the motion appears very irregular and quite indistinguishable from motion 

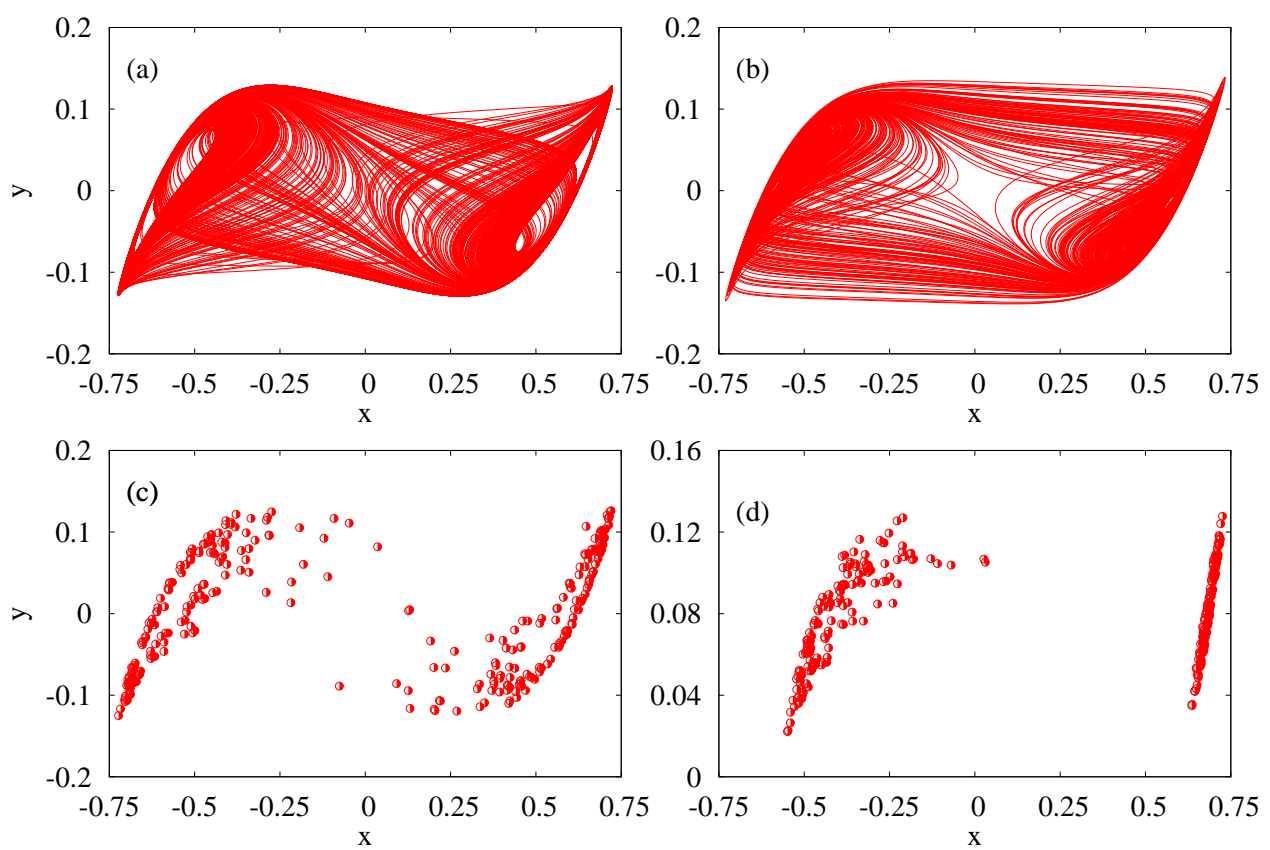

Fig. 4 Attractors for different $a_{0}$ and for the parameters $\alpha=0.35, \beta=300, m=100, \gamma=0.2, \mu=1.0$, and $\omega=10$. (a) Phase portrait of a double-scroll chaos for $a_{0}=0$, (b) phase portrait of a hyperchaotic attractor for $a_{0}=1.51$, (c) Poincaré section $a_{0}=1.72$ and (d) Poincaré section for $a_{0}=1.51$ depicting attractor splitting in the hyperchaotic state.

on a chaotic attractor and for $t \geq 580$, the system is in a stable quasiperiodic motion. Inside this narrow parameter window, attractors may co-exist and the length of time during which the orbit spends in the initial chaotic phase depends on the value $a_{0}$ as well as the initial conditions. For instance, for $a_{0}=1.78$, the initial transient phase is longer, $t \approx 950$.

With increasing $a_{0}$, a different scenario is observed. Fig. 6 shows the enlarged portion of Fig. 2 in the regime III. Here, we find multiple transitions beginning with a sudden loss of stability by the limit cycle in the broad range of the driving amplitude, namely $1.8 \leq a_{0} \leq 7.8$ (region II) and the appearance of alternating periodic orbits, first a period-10 attractor (shown in Fig. 7(a) for $a_{0}=7.836$ together with a limit cycle at $a_{0}=7.5$ ), followed by various orbits of higher periodicity (typically $n>10$, where $n$ is the period of the orbit) spanning the entire regime $7.8 \leq a_{0} \leq 9.5$. This sequence is however terminated with the emergence of a chaotic band in the range $9.13 \leq a_{0} \leq 9.58$ where $\lambda_{1}$ is typically positive. A typical chaotic attractor in this band for $a_{0}=9.45$ is shown in Fig. 7(b). Following the chaotic band, a period-17 orbit (See Fig 7(c)) born in the large window loses its stability in a bifurcation at $a_{0} \approx 9.92$ with the birth of a strange nonchaotic attractor of quasiperiodic orbits exemplified by the orbits in Fig $7(\mathrm{~d})$ for $a_{0}=9.95$. Notice that the strange nonchaotic attractor in Fig 7(d) has structural resemblance with the chaotic attractor in Fig 7(b). However, the attractor in Fig 7(b) has one positive Lyapunov exponent $\left(\lambda_{1}=0.212\right)$ and one negative $\left(\lambda_{2}=-0.706\right)$, whereas $\lambda_{1}=0$, and $\lambda_{2}=-0.479$ for the attractor in Fig $7(\mathrm{~d})$. As $a_{0}$ increases further pass $a_{0} \approx 10.05$, we find that the region $10.1 \leq a_{0} \leq 10.35$ is dominated by quasiperiodic orbits of invariant curve, which undergoes Hopf bifurcation to a period-1 orbit. Deformation and lost of smoothness of the invariant curve takes place around $a_{0} \approx 10.0$ before its final destruction as $a_{0}$ decreased -giving rise to the strange nonchaotic attractor.

\section{Global Bifurcation Structure}

In the previous section, we examined the local bifurcations with $a_{0}$ being the bifurcation parameter. The bifurcations may also be investigated using $\omega$ as the bifurcation parameter. However, a global view of this system can conveniently be captured by simultaneously scanning a wide range of the forcing parameters, i.e. bifurcations in $a_{0}-\omega$ parameter space plane. Here, we employ the Lyapunov spectrum formed by all the Lyapunov exponents $\lambda_{n}(i=1,2,3,4)$ as a tool for constructing the dynamical system parameter space diagram. The nature of the attractor can be characterized by the values of these four Lyapunov exponents. The system is considered hyperchaotic (HC), if $\lambda_{1,2}>0, \lambda_{3,4} \leq 0$, chaotic $(\mathrm{CH})$ if $\lambda_{1}>0, \lambda_{2,3,4} \leq 0$, quasi-periodic oscillation on 


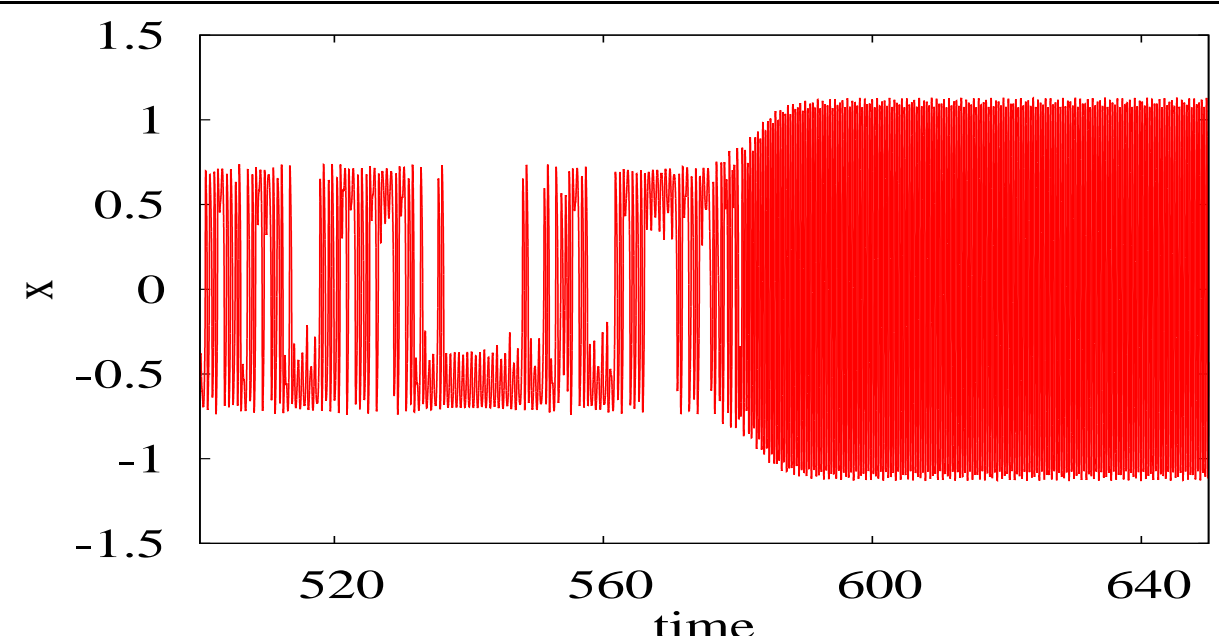

Fig. 5 Time Series of a transient chaos for $a_{0}=1.77$. Other parameters are: $\alpha=0.35, \beta=300, m=100, \gamma=0.2, \mu=1.0$, and $\omega=10$. The initial phase is $t \leq 580$.

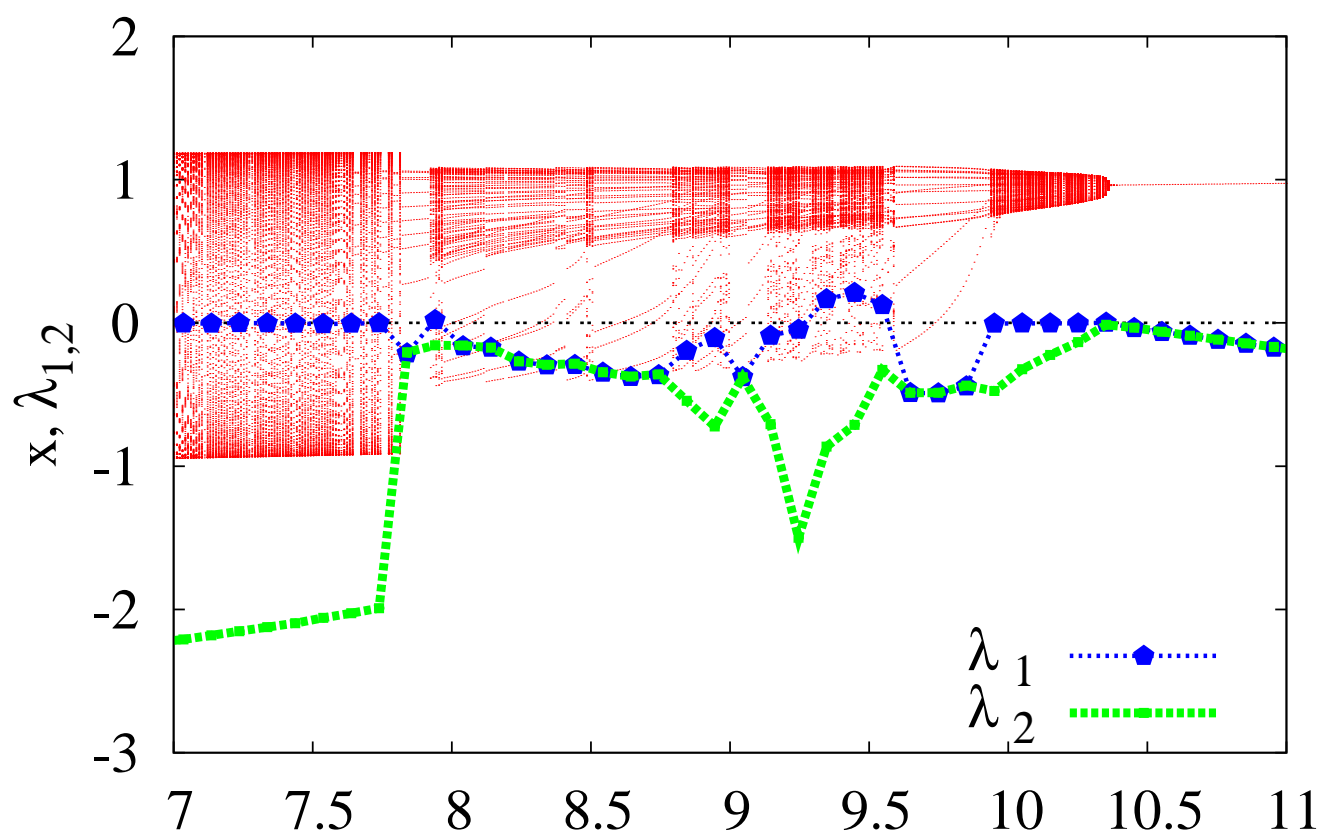

Fig. 6 Enlarged bifurcation diagram of the local maximum of $x$ an $\mathrm{a}_{(}$the corresponding Lyapunov exponents as function of the driving amplitude, for region III in Figure 2 for the same set of parameters.

a torus $T^{2}$ or limit cycle (QP), if $\lambda_{1}=0, \lambda_{2,3,4} \leq 0$; and periodic orbit or a fixed point $(\mathrm{PO})$ when $\lambda_{i}<0(i=$ $1,2,3,4)$. Keeping the above characteristic properties, and with the aid of GramSchmidt orthonormalization, we computed all the Lyapunov exponents by applying the Wolf et al.'s algorithm [42] as done previously. We remark that due to the non-exact computation of the exponents, we consider an exponent null if its value is within the interval $-0.0005 \leq \lambda \leq 0$.

Shown in Fig. 8 are typical two-parameter space plots showing clearly different regions of hyperchaos
$(\mathrm{HC})$, chaos $(\mathrm{CH})$, quasiperiodic $(\mathrm{QP})$ orbits and periodic orbits $(\mathrm{PO})$. For $\mu=1.0$ on the left panel, both hyperchaotic and chaotic orbits can show up by making appropriate choice of the external forcing parameters. In the low frequency regime, $\omega<3$, the dynamics are largely dominated by $\mathrm{HC}$ and $\mathrm{CH}$ orbits for nearly all forcing amplitudes, $a_{0}$. As the frequency increases pass $\omega \approx 3.0$, QP and $\mathrm{PO}$ orbits begin to appear, dominating a wide range of the parameter space. For $\omega=10$, we find that $\mathrm{HC}$ orbits lies within the low amplitude 

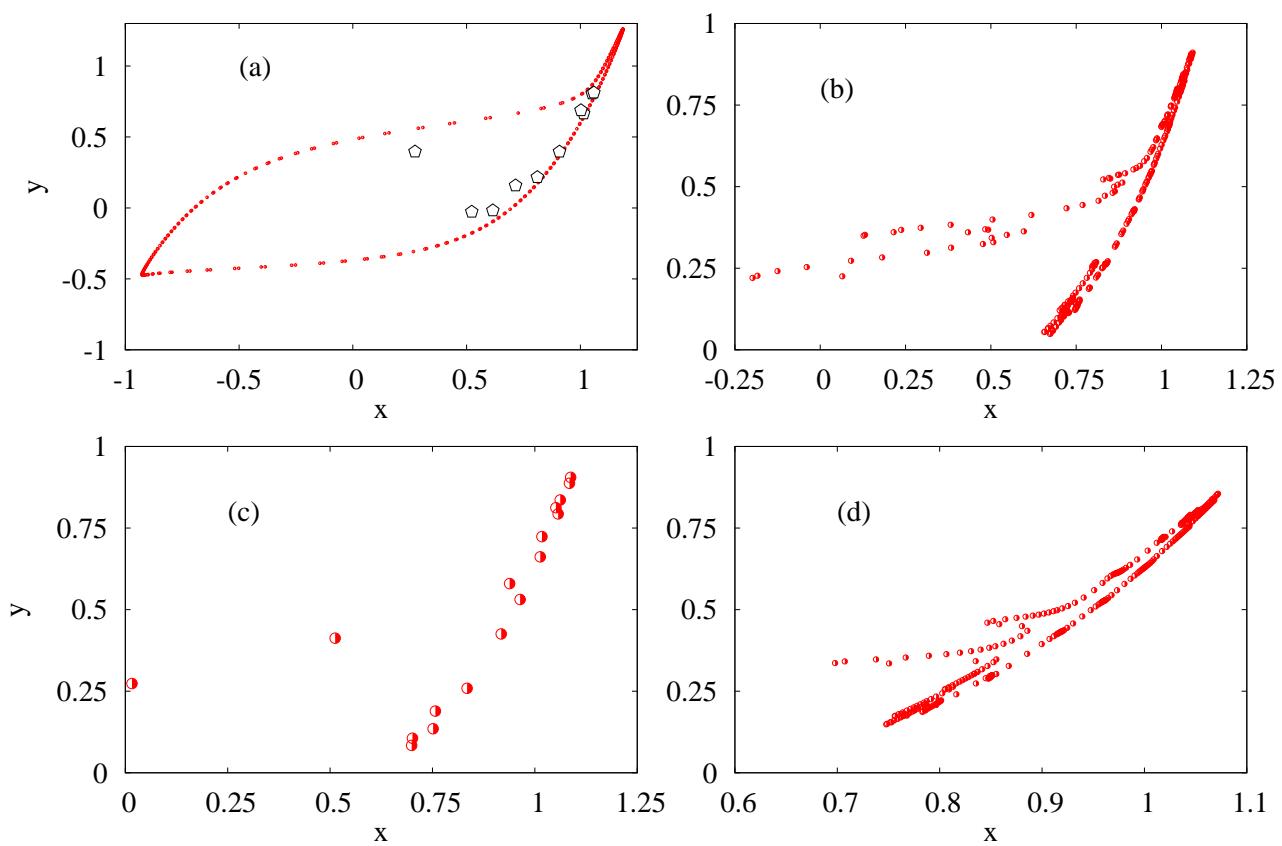

Fig. 7 Poincaré sections showing various attractors during the transitions from regions II to IV of Fig. 2 for the same set of parameters and for increasing $a_{0}$. (a) Limit cycle (closed dotted curve) for $a_{0}=7.5$ together with period-10 orbit (open points) for $a_{0}=7.836$ (Note that there are two repeated overlapping points), (b) new chaotic attractor for $a_{0}=9.45$, (c) period-17 orbit for $a_{0}=9.745$ and (d) strange non-chaotic attractor for $a_{0}=9.95$.

regime, typical $a_{0}<2.0$; while higher values of $a_{0}$ will drive the system to $\mathrm{QP}$ states.

For further increase in $\omega$ up-to $\omega \approx 17.0$, we observe phase locking at $\omega=\omega_{0} \approx 17.0$, where the harmonic force locks with oscillations of the system - the consequence being stabilization to periodic state. The periodic state is a stable stationary solution in the parameter space (i.e. mode locking regions) where the frequency of the oscillator coincides with the forcing frequency. This region, called synchronization region, or resonance (or Arnold) tongue occurs when a self-excited oscillation interacts with a driving force, resulting in an adjustment of the oscillation. [12,32].

Finally, it would be significant to examine the effects of the parallel resistance, $R_{p}$ in Fig. 1 denoted by the dimensionless quantity, $\mu$ in eqs. 4 and 5. In Fig. 8(b), we illustrate the effect of $\mu$ on the global dynamics of the driven oscillator. We find that for $\mu=1.5$, chaotic $(\mathrm{CH})$ and hyperchaotic $(\mathrm{HC})$ regions are replaced by periodic orbits - Implying that the parallel resistance could act as a simple control input in the system. The parameter space is largely dominated by periodic orbits with some visible regions of chaos and hyperchaos behaviour.

\section{Conclusions}

In this paper, we have reported on the influence of an external periodic signal on the familiar double-scroll chaotic attractor of the autonomous van der-Pol Duffing oscillator circuit by introducing to the circuit a periodic signal source. As the external forcing parameters are varied, new dynamical behaviors emerge, including hyperchaos arising from attractor splitting, quasiperiodicity, strange-nonchaotic attractors and periodic orbits of higher periodicity. The regimes of existence of different dynamical behaviors in two-parameter space of the driving force were identified with the aid of two parameter bifurcation diagram computed using complete spectra of the Lyapunov exponents. By comparison, we find that the modified model presented by Fotsin et al. $[11,10,9]$ show more complex behavior when periodically driven than the Matouk model $[27,26]$. For instance, in a wide range of $\omega-a_{0}$ parameter space, adjusting the value of the parallel resistance $R_{p}$ in the circuit of Fig. 1, denoted by the dimensionless parameter $\mu$, in eq. 5 , drives the system to periodic states - implying that variation in $R_{p}$ would initiate chaos control in the systems. Thus, chaotic behaviour could be conveniently tamed in practical experiment, whereby $R_{p}$ act as a simple limiter. This is very important for practical applications where chaos is undesirable. On the other hand, when $R_{p}$ is absent, that is $\mu=1$, the system 

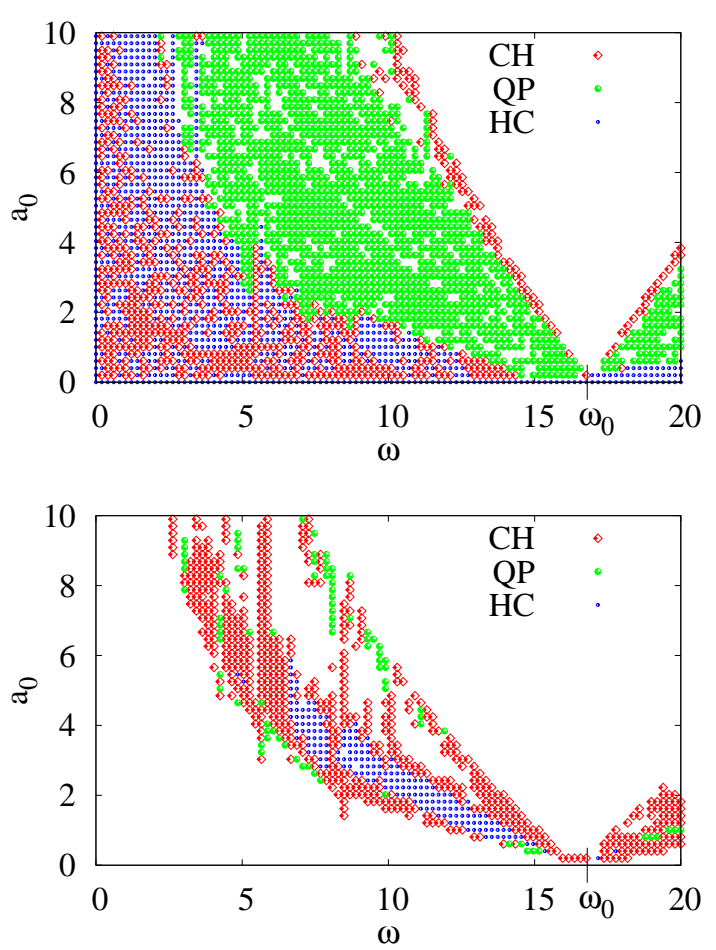

Fig. 8 Two Parameter space diagram obtained from the Lyapunov spectra, indicating the behavior of the driven van der Pol-Duffing circuit for $\alpha=0.35, \beta=300, m=100, \gamma=0.2$, and $\mu=1.0$, showing different regions of hyperchaos (dotted blue), chaos (dotted red), quasiperiodic (dotted green) and periodic (elsewhere - white) orbits. In (a) $\mu=1.0$ and (b) $\mu=1.5$.

would be more complex in the presence of the driving signal, presenting also chaos and hyperchaos in larger parameter space. In communication systems where security is important, such high complexities are significant for secure communication applications.

\section{Acknowledgments}

UEV is supported by the Royal Society of London through the Newton International Fellowship Alumni Scheme. $\mathrm{BRN}$ is grateful to Brazilian Government (CNPq) for financial support within the project $\mathrm{CNPq} / \mathrm{PROAFRICA}$ 490265/2010-3. We acknowledge the comments of reviewers.

\section{References}

1. A. Y. Aguilar-Bustos, C. Cruz-Hernández, R. M. LópezGutirrez, E. Tlelo-Cuautle, and C. Posadas-Castillo. Emergent Web Intelligence: Advanced Information Retrieval. Advanced Information and Knowledge Processing. Springer, London, 2010.
2. M. S. Baptista and I. L. Caldas. Phase-locking and bifurcations of the sinusoidally-driven double scroll circuit. Nonlin. Dynam., 17:119139, 1998.

3. D. C. Braga, L. F. Mello, and M. Messias. Bifurcation analysis of a Van der Pol-Duffing circuit with parallel resistor. Math. Prob. Engin., 2009:1-26, 2009.

4. A. Cenys, A. Tama s̃evicius, A. Baziliauskas, R. Krivickas, and E. Lindberg. Hyperchaos in coupled Colpitts oscillators. Chaos, Solitons \& Fractals, 17:349-353, 2003.

5. L. O. Chua, L. J. Kocarev, K. Eckert, and M. Itoh. Experimental chaos synchronization in Chua's circuit. Int. J. Bifurc. Chaos, 2:705-708, 1992.

6. F. Q. Dou, J. A. Sun, and W. S. Duan. Antisynchronization in different hyperchaotic systems. Commun. Theor. Phys., 50:907, 2008.

7. A. S. Elwakil and M. P. Kennedy. Construction of classes of circuit independent chaotic oscillators using passiveonly nonlinear. IEEE Trans. Circ. and Syst., 48:289-307, 2001.

8. A. S. Elwakil and A. M. Soliman. A family of Wien-type oscillators modified for chaos. Int. J. Circ. Theor. Appl., 25:561-579, 1997.

9. G. J. Fodjouong, H. B. Fotsin, and P. Woafo. Synchronizing modified Van der Pol-Duffing oscillators with offset terms using observer design: application to secure communications. Phys. Scripta, 75(5):638-644, 2007.

10. H. Fotsin, S. Bowong, and J. Daafouz. Adaptive synchronization of two chaotic systems consisting of modified Van der Pol-Duffing and Chua oscillators. Chaos, Solitons \&f Fractals, 26(1):215-229, 2005.

11. H. B. Fotsin and P. Woafo. Adaptive synchronization of a modified and uncertain chaotic Van der Pol-Duffing oscillator based on parameter identification. Chaos, Solitons E Fractals, 24(5):1363-1371, 2005.

12. D. García-Álvarez, A. Stefanovska, and P. V. E. McClintock. High-order synchronization, transitions, and competition among arnold tongues in a rotator under harmonic forcing. Phys. Rev. E, 77:056203, 2008.

13. M. G. M. Gomes and G. P. King. Bistable chaos. II. Unfolding the cusp. Phys. Rev. A, 46:3100-31099, 1992.

14. C. Grebogi, E. Ott, and J. A Yorke. Chaotic attractors in crisis. Phys. Rev. Lett., 48:1507-1510, 1982.

15. C. Grebogi, E. Ott, and J. A Yorke. Crises, sudden changes in chaotic attractors and transient chaos. Physica $D, 7: 181-200,1983$.

16. K. Grygiel and P. Szlachetka. Hyperchaos in secondharmonic generation of light. Opt. Commun., 158:112118, 1998.

17. C. Hayashi. Nonlinear oscillations in physical systems. McGraw-Hill, New York, 1964.

18. F. M. Moukam Kakmeni, S. Bowong, C. Tchawoua, and E. Kaptouom. Strange attractors and chaos control in a Duffing - Van der Pol oscillator with two external periodic forces. J. Sound Vibr., 277:783, 2004.

19. G. P. King and S. T. Gaito. Bistable chaos. I. Unfolding the cusp. Phys. Rev. A, 46:3092-3099, 1992.

20. X. Li, J. C. Ji, C. H. Hansen, and C. Tan. The response of a Duffing-van der Pol oscillator under delayed feedback control. J. Sound Vibr., 291(3-5):644-655, 2006.

21. N. H. Liao and Z. H. Hu. A hybrid secure communication method based on synchronization of hyper-chaos systems. In G. Tomar, G. S. Mittal, and Z. Frank, editors, 2012 International Conference on Communication Systems and Network Technologies, CSNT, volume 2012, pages 289-293, Rajkot, Gujrat, India, May 2012. IEEE Computer Society, IEEE Computer Society Conference Publishing Services. 
22. Y. Liu, Q. Yang, and G. Pang. A hyperchaotic system from the Rabinovich system. Journal of Computational and Applied Mathematics, 234:101-113, 2010.

23. J. Ma, A. B. Li, Z. S. Pu, L. J. Yang, and Y. Z. Wang. A time-varying hyperchaotic system and its realization in circuit. Nonlin. Dynam., 62:535-541, 2010.

24. R. A. Madan. Chua's Circuit: A paradigm for Chaos. World Scientific, Singapore, 1993.

25. G. M. Maggio, O. D. Feo, and M. P Kennedy. Nonlinear analysis of the colpitts oscillator and applications to design. IEEE Trans. Circ. and Syst., 46:1118-1130, 1999.

26. A. E. Matouk. Chaos, feedback control and synchronization of a fractional-order modified autonomous Van der Pol-Duffing circuit. Commun. Nonlinear. Sci. Numer. Simulat. 16 (2011) 975986, 16:975-896, 2011.

27. A. E. Matouk and H. N. Agiza. Bifurcations, chaos and synchronization in ADVP circuit with parallel resistor. J. Math. Anal. Appl., 341:259-269, 2008.

28. K. Murali, M. Lakshmanan, and L. O. Chua. Bifurcation and chaos in the simplest dissipative non-autonomous circuit. Intern. J. Bifurc. Chaos, 4(6):1511-1524, 1994.

29. E. Ott. Chaos in Dynamical Systems. Cambridge University Press, Cambridge, 2002.

30. F. Prebianca, H. A. Albuquerque, and R. M Rubinger. On the effect of a parallel resistor in the Chua's circuit. In Dr Elbert Macau, editor, J. Phys., volume 285 of Conf. Series, page 012005. Dynamics Days South America 2010 International Conference on Chaos and Nonlinear Dynamics 26-30 July 2010, Institude of Physics, Europe, 2011.

31. O. E. Rössler. An equation for hyperchaos. Phys. Lett. A, 71(2-3):155-157, 1979.

32. W. D. Suranga Ruhunusiri and J. Goree. Synchronization mechanism and arnold tongues for dust density waves. Phys. Rev. E, 85:046401, 2012.

33. I. Sliwa, K. Grygiel, and P. Szlachetka. Hyperchaotic beats and their collapse to the quasiperiodic oscillations. Nonlinear Dyn, 53:13-18, 2008.

34. N. Smaoui, A. Karouma, and M. Zribib. Secure communications based on the synchronization of the hyperchaotic chen and the unified chaotic systems. Phys. Scripta, 16:3279-3293, 2011.

35. J. C. Sprott. A new class of chaotic circuits. Phys. Lett. A, 266:19-23, 2000.

36. T. Stankovski, A. Duggento, P. V. E. McClintock, and A. Stefanovska. Inference of time-evolving coupled dynamical systems in the presence of noise. Phys. Rev. Lett., 109:024101, 2012.

37. K. Sun, X. Liu, C. Zhu, and J.C. Sprott. Hyperchaos and hyperchaos control of the sinusoidally forced simplified Lorenz system. Nonlin. Dynam., 69:1383-1391, 2012.

38. R. Tchitnga, H. B. Fotsin, B. Nana, P. H. LouodopFotso, and P. Woafo. Hartleys oscillator: The simplest chaotic two-component circuit. Chaos, Solitons \& Fractals, 45:306313, 2012.

39. K. Thamilmaran, M. Lakshmanan, and K. Murali. Rich variety of bifurcations and chaos in a variant of MuraliLakshmanan-Chua circuit. Intern. J. Bifurc. Chaos, 10:1781-1785, 1994.

40. Y. Ueda. The Road to Chaos - II. Future University Press, Hakodate, 2001.

41. U. E. Vincent, R. K. Odunaike, J. A. Laoye, and A. A. Gbindinninuola. Adaptive backstepping control and synchronization of a modified and chaotic Van der PolDuffing oscillator. J. Control Theory Appl, 9(2):141-145, 2011.
42. A. Wolf, J. B. Swift, H. L. Swinney, and J. A. Vastano. Determining Lyapunov exponents from a time series. Physica D, 16:285-17, 1985.

43. S. Yu, J. Lu, and G. Chen. A family of n-scroll hyperchaotic attractors and their realization. Phys. Lett. A, 364:244-251, 2007.

44. X. Zhou, B. Kong, and H. Ding. Synchronization and anti-synchronization of a new hyperchaotic 1 system with uncertain parameters via the passive control technique. Phys. Scripta, 85:065004, 2012. 